\title{
DISEÑO Y VALIDACIÓN DE UN PROTOCOLO DE ATENCIÓN PSICOLÓGICA PARA LA IDEACIÓN SUICIDA
}

\author{
Design AND VAlidation of A PSYCHOlogicAl ATtention Protocol For \\ SUICIDAL IDEATION
}

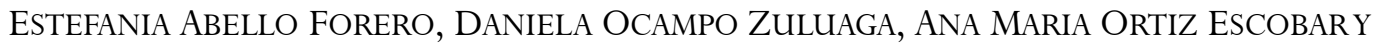 \\ JORGE OSWALDO GONZALEZ ORTIZ ${ }^{1}$ \\ UNIVERSIDAD DE SAN BUENAVENTURA, SEDE BOGOTÁ
}

FECHA RECEPCIÓN: 11/05/2020 • FECHA ACEPTACIÓN: 1/10/2020

Para citar este artículo: Abello, E., Ocampo, D., \& González, J., (2020). Diseño y validación de un Protocolo de Atención Psicológica para la Ideación Suicida. Psychologia, 14(2), 61-68. doi:10.21500/19002386.4824.

\section{Resumen}

La cifra de personas que presentan o han presentado alguna conducta suicida ha aumentado significativamente en Colombia desde el año 2014 al 2019, convirtiendo la conducta suicida en una problemática de salud pública; sin embargo, es un concepto amplio, que abarca diferentes tipos de conductas, por esto es necesario establecer un protocolo de atención para estandarizar las acciones del terapeuta o personas que se desempeñan en los servicios prestadores de salud. El presente artículo tiene como objetivo describir cómo se realizó el diseño y la validación de un protocolo de atención psicológica para atención en ideación suicida de los consultantes que asisten a un centro de atención psicológica de una institución universitaria de la ciudad de Bogotá. Se realizó un estudio cuantitativo, de tipo instrumental, el cual implicó: a) el diseño de la ruta de atención, situaciones que activan el protocolo y el módulo de atención en crisis; b) la participación de 10 jueces expertos con el fin de realizar la validación por contenido de todos los componentes del protocolo de atención psicológica. Los resultados arrojaron que el diseño es pertinente, suficiente y relevante para la problemática que se buscó abordar.

Palabras clave: Conducta suicida, protocolo de atención psicológica.

\begin{abstract} significantly in Colombia from 2014 to 2019, making suicidal behavior a public health problem. However, it is a

1 jgonzalez@usbbog.edu.co

Currently, the number of people who present or have presented some suicidal behavior has increased 
broad concept that covers different types of behavior, so it is necessary to establish a protocol of care to standardize the actions of the therapist and/or people who work in health care services. The present article aims to describe how a psychological care protocol was designed and validated for the care of suicidal ideation of consultants who attend a psychological care center at a university institution in the city of Bogotá. A quantitative, instrumental study was carried out, which involved: a) designing the care route, situations that activate the protocol and the crisis care module; b) the participation of 10 expert judges to carry out the validation of the content of all the components of the psychological care protocol. The results showed that the design of the care protocol is pertinent, sufficient, and relevant to the problem to be addressed.

Keywords: Suicidal behavior, protocol of attention, suicidal ideation, Psychological Care Center.

\section{Introducción}

De acuerdo con la Ley 1122 de 2007 de Colombia, la salud pública está constituida por un conjunto de políticas que buscan garantizar de manera íntegra la salud de la población, por medio de acciones dirigidas de manera individual y colectiva, haciendo de sus resultados indicadores de las condiciones de vida, bienestar y desarrollo. Dichas acciones se realizan bajo la rectoría del Estado y deberán promover la participación responsable de todos los sectores de la comunidad (Ministerio de Salud y Protección Social, 2018).

Una función esencial relacionada con la responsabilidad estatal y ciudadana para la protección de la salud es la vigilancia en salud pública, la cual consiste en el proceso sistemático y constante de recolección, análisis, interpretación y divulgación de datos específicos, relacionados con la salud para su utilización en la planificación, ejecución y evaluación de la práctica en salud pública, la cual depende de una red de actores que conforman el Sistema de Vigilancia de Salud Pública, encabezado por el Ministerio de Salud y Protección Social en el Grupo de Vigilancia de la Dirección de Epidemiología y Demografía (Ministerio de Salud y Protección Social, 2018).

Con relación a lo anterior, la epidemiología es la herramienta básica de la salud pública (Bonita, Beaglehole \& Kjellstróm, 2008), y se define como el estudio de la distribución y los determinantes de estados o eventos, en general de enfermedades, relacionados con la salud y aplicación de esos estudios al control de enfermedades y otros problemas de salud (Organización Mundial de la Salud, 2018).

Los códigos CIE-10 relacionados con la conducta suicida son: X60 al X84. Siempre que se atienda este evento en los servicios de salud, se recomienda realizar una evaluación completa del estado de salud mental con el fin de identificar probables trastornos mentales asociados (Códigos CIE-10 F00 a F99) y, asimismo, otras circunstancias psicosociales que en buena parte de los casos también se encuentran presentes (Códigos CIE-10 Z55 a Z65), los cuales deben ser consignados como diagnósticos relacionados en el Registro Individual de Prestación de Servicios de Salud (RIPS) y en historia clínica (Ministerio de Salud, 2018, p. 2).

La conducta suicida presenta en la actualidad un aumento de la mortalidad en la población. De acuerdo con la Guía de Práctica Clínica (GPC) Número 60, esta consiste en un:

(...) proceso que hace referencia a los pensamientos o conductas que llevan al acto de quitarse la vida intencionalmente. Puede clasificarse en tres categorías: ideación, plan e intento suicida. La ideación, abarca los pensamientos del acto suicida, pero sin una planeación sobre el mismo; en el plan, estos pensamientos son más elaborados e integran un método para llevar a cabo el acto suicida; en el intento, existe un comportamiento potencialmente autolesivo en el que se pueden presentar diferentes grados en la intención de morir. (2017, p. 21)

El proceso finaliza con el suicidio consumado, que se entiende como:

(...) la muerte derivada de la utilización de cualquier método (envenenamiento, ahorcamiento, herida por arma de fuego o cortopunzante, lanzamiento al vacío, a un vehículo o cualquier otra forma), con evidencia, explícita o implícita, de que fue autoinfligida y con la intención de provocar 
el propio fallecimiento. (Ministerio de Salud, 2018, p. 3)

Los datos epidemiológicos de la conducta suicida en Colombia reportaron 36163 casos, en promedio, entre 2009 y 2018. Según el Ministerio de Salud, en el año 2017 la tasa de suicidio se incrementó de 4,5\% a 5,7 \% (25,1\%); el 81,5\% fueron hombres; la mayoría de estos, entre los 20 y los 24 años de edad; es decir, se suicidan 5 hombres por cada mujer; las mayores tasas se presentaron en adultos mayores (18 por cada 100000 habitante/año) y en adultos jóvenes (13 por cada 100000 habitante/año). Igualmente, las estadísticas indican que se reportaron más casos de suicidios en la cabecera municipal (74\%); los meses con más reportes de suicidios son: mayo (253 casos) y julio (232 casos); en cuanto a los días con mayor presencia son los domingos (485 casos) y los lunes (419 casos). Las estadísticas colombianas afirman que las técnicas predominantes para quitarse la vida son: asfixia (ahorcamiento) (64,2 \%), uso de tóxicos (16,71\%) y uso de proyectil de arma de fuego $(10,93 \%)$. Entre las posibles razones se encontró: conflicto pareja-desamor: $41 \%$; enfermedad física/mental: 29 \% y económicas: 12 \%; el 77 \% ocurrió en la vivienda. Según departamento y municipio: Arauca y Vaupés presentaron las mayores tasas de suicidio: 16/100 000 habitante/año; Puerto Arica (Amazonas) 92/100 000 habitante/año; Bogotá tuvo una tasa de 4,6 cada 100000 habitante/año) (Instituto Nacional de Medicina Legal y Ciencias Forensis, 2018).

La ideación suicida por regiones muestra que es más alta en Bogotá (10,3\%); no se encontraron diferencias en los adultos en situación de pobreza comparados con quienes no lo están. Según la escolaridad, la proporción más alta de ideación se encontró en personas con secundaria completa $(7,3 \%)$. Alrededor de un tercio $(35,9 \%)$ de las personas que piensan suicidarse han tenido planes e intentos $(39,2 \%)$. Los intentos suicidas son más frecuentes en mujeres (3,3\%) que en hombres $(1,9 \%)$, de los cuales alrededor de la mitad de las personas que han intentado suicidarse lo describen como un intento serio. Un cuarto de las mujeres que intentan suicidarse lo hacen como una manera de pedir ayuda (Encuesta Nacional de Salud Mental, 2015).

Teniendo en cuenta el impacto que tiene la conducta suicida en la salud pública, la epidemiología y el uso adecuado de los diferentes métodos y técnicas para asegurar un desempeño apropiado en esta área, se hace uso de protocolos clínicos que tienen el fin de estandarizar la calidad en la atención de salud y ayudan al clínico a tomar decisiones en momentos de incertidumbre. Los protocolos, además de ser herramientas que contribuyen a normalizar la práctica, son una fuente poderosa de información al tiempo que facilita la atención a personal de nueva incorporación. El objetivo que se espera cumplir al elaborar un protocolo es disminuir la variabilidad en la práctica y guiar hacia un mismo centro, por lo que la estructura debe ser normalizada (Sánchez et al., 2011).

Para la elaboración de estos protocolos es necesario utilizar modelos de referencia para así disminuir errores y defectos previos haciendo que se logre adquirir calidad en cuanto a estructura y homogeneidad de los procedimientos, y del resultado final. Es fundamental entender que este proceso debe cumplir fases que incluyen la definición de la problemática a resolver, la elaboración de un documento que recoja los conocimientos previos, contar con fases de pilotaje, análisis e implementación y, finalmente, contar con fases de monitoreo periódicas que fomenten los ajustes y actualizaciones del protocolo (Saura \& Saturno, 1996).

Por su parte, el National Health Services (por sus siglas en inglés, NHS) creó una guía de elaboración de protocolos de acuerdo con los lineamientos básicos de NICE (National Institute for Health and Care Excellence) de 12 pasos, en los cuales contempla la selección y priorización del tema a tratar, organización del equipo de trabajo, involucramiento de pacientes y usuarios, creación de conciencia y compromiso, recolección de información, establecimiento de la línea base, realización del protocolo, pilotaje, implementación, monitoreo de los cambios y revisión del protocolo; todo lo anterior, con el propósito de tener un protocolo adecuado con las necesidades de la institución universitaria que brinda atención psicológica, en problemáticas como la conducta suicida y que responda a los lineamientos de las guías de práctica clínica adaptadas en el país (Guía de Práctica Clínica Número 60, 2017).

Teniendo en cuenta la GPC de conducta suicida que brinda los lineamientos a nivel nacional y con el fin de disponer de una ruta en el Centro de Atención Psicológica (CAP), se elaboró el presente protocolo de atención. 
Consideraciones éticas

De acuerdo con la Ley 1090 de 2006, por la cual se reglamenta el ejercicio de la profesión de psicología, se dicta el código Deontológico y Bioético y otras disposiciones, al cual se acogen los profesionales en psicología, el Artículo $2^{\circ}$, 5. Se mantendrá la confidencialidad de la información obtenida de los consultantes atendidos en el CAP y no se revelará información salvo con el consentimiento informado de la persona o del representante legal, excepto en aquellas situaciones particulares en que no hacerlo llevaría a un evidente daño a la persona y a otros (Ministerio de la Protección Social, 2006).

El Artículo $13^{\circ}$, como regla de conducta profesional, proporciona principios generales que ayudan a tomar decisiones informadas en la mayor parte de las situaciones en el sitio designado, fundamentado en los principios de beneficencia, no-maleficencia, autonomía, justicia, veracidad, solidaridad, lealtad y fidelidad; principios en los que este protocolo se basa (Ministerio de la Protección Social, 2006).

De acuerdo con la Resolución 8430 de 1993, por la cual se establecen las normas científicas, técnicas y administrativas para la investigación en salud, en los artículos 10 y 11 se clasifica la presente investigación sin riesgo, ya que es un estudio que emplea técnicas y métodos de investigación retrospectivos y aquellos en los que no se realiza ninguna intervención o modificación intencionada de las variables biológicas, fisiológicas, psicológicas o sociales de los individuos que participan en el estudio (Resolución número 8430, 1993).

\section{Método}

Tipo de estudio

Se realizó un estudio cuantitativo, de tipo instrumental. Este tipo de estudios engloba aquellos trabajos encaminados a desarrollar algún tipo de material en pruebas o instrumentos (Montero \& León, 2007). Asimismo, se siguieron los lineamientos del NHS (2014) para el diseño del protocolo, y para la validación se realizó un procedimiento de evaluación con la participación de 10 jueces expertos, utilizando el coeficiente de razón de validez por contenido según los criterios de Lawshe (1975).
Participantes

Dado que el estudio no contempló la aplicación a pacientes o consultantes, los participantes corresponden a 10 jueces profesionales de la psicología, expertos en validación de instrumentos, en psicología clínica, con experiencia en conducta suicida y en intervención en crisis.

\section{Instrumentos}

Se diseñó un Formato de Calificación y Validación por Juicio de Expertos, mediante el cual se evaluaron los componentes del protocolo de atención psicológica en ideación suicida (protocolo de activación, ruta de atención, módulo de atención en crisis y módulo de atención psicológica).

Criterios de calificación: a) suficiencia, entendiéndose como que el protocolo tiene la capacidad de abordar situaciones de emergencia y atención a la ideación suicida; b) claridad, entendiéndose como la facilidad de comprensión teniendo en cuenta la adecuada sintáctica y semántica; c) pertinencia, entendiéndose como la relación lógica con la problemática a tratar, y d) relevancia, entendiéndose como la importancia del componente dentro del protocolo. Cada uno de los componentes se califica entre 1 y 4 , donde 1 no cumple el criterio y 4 cumple con un alto nivel.

\section{Procedimiento}

Fase 1. Se realizó la revisión de la literatura con el fin de identificar los contenidos pertinentes para la realización del protocolo y establecer el marco teórico. De igual manera, y de acuerdo con la guía de la NHS (2014), se realizó la definición y priorización del tema; se estableció el equipo de trabajo y sus funciones; y se establecieron objetivos y alcances del protocolo.

Fase 2. Se diseñó la ruta de atención psicológica para conducta suicida y los componentes del protocolo (protocolo de activación, módulo de atención en crisis y módulo de atención psicológica).

Fase 3. Se realizó la validación por jueces y la revisión y adecuación del protocolo de acuerdo con las sugerencias de los jueces. 


\section{Resultados}

Se presentan los resultados obtenidos durante el diseño y la validación por jueces del protocolo.

Fase de Diseño del protocolo: A continuación, se enuncian los 4 componentes que constituyen el protocolo.

- Protocolo de activación: Se describen dos situaciones por las cuales un consultante puede llegar al Centro de Atención Psicológica Universitaria reportando conducta suicida:

a. Evento crónico: en donde el consultante ya ha solicitado una cita con alguna de las especialidades en el Centro; por vía telefónica o presencial (ver Figura 1).

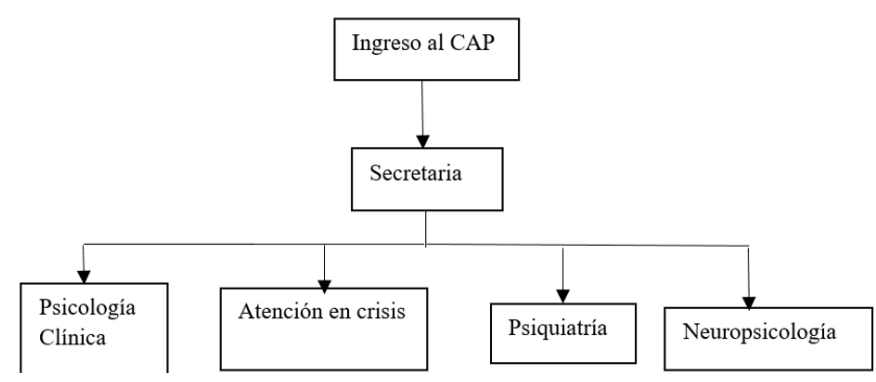

Figura 1. Ingreso al Centro de Atención Psicológica Universitaria por evento crónico

b. Evento agudo: (Ver Figura 2) se recibe al consultante en el centro de atención universitaria, mediante remisión de alguna institución de salud, educativa u otra, donde el motivo de consulta haga referencia a la conducta suicida.

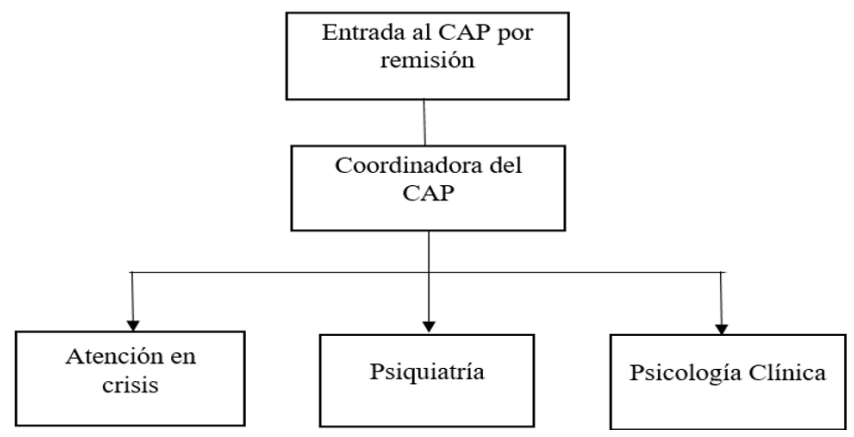

Figura 2. Ingreso al Centro de Atención Universitario por evento agudo

-Ruta de atención psicológica para la conducta suicida: después de clasificar y activar el protocolo, se realiza el ingreso habitual del consultante por portería hacia la recepción, donde el consultante realiza el diligenciamiento de datos personales y pago del servicio de consulta. Se conduce al consultante al triage en caso de ser nuevo y presentarse sin cita para hacer la clasificación de estado de crisis; si se encuentra riesgo de intento suicida, se remite a una institución de atención médica de urgencias, en caso contrario se continúa con el proceso de consulta, se activa el protocolo de atención en conducta suicida. Si se reporta ideación o plan suicida por parte del consultante en la entrevista inicial con el terapeuta, se continúa con los módulo de atención, los cuales incluyen la aplicación de pruebas psicológicas: Escala de Depresión Montgomery-Asberg (MADRS), Escala de Desesperanza de Beck (BHS) y Escala de Ideación Suicida de Beck (ISB) (Ver Figura 3). 


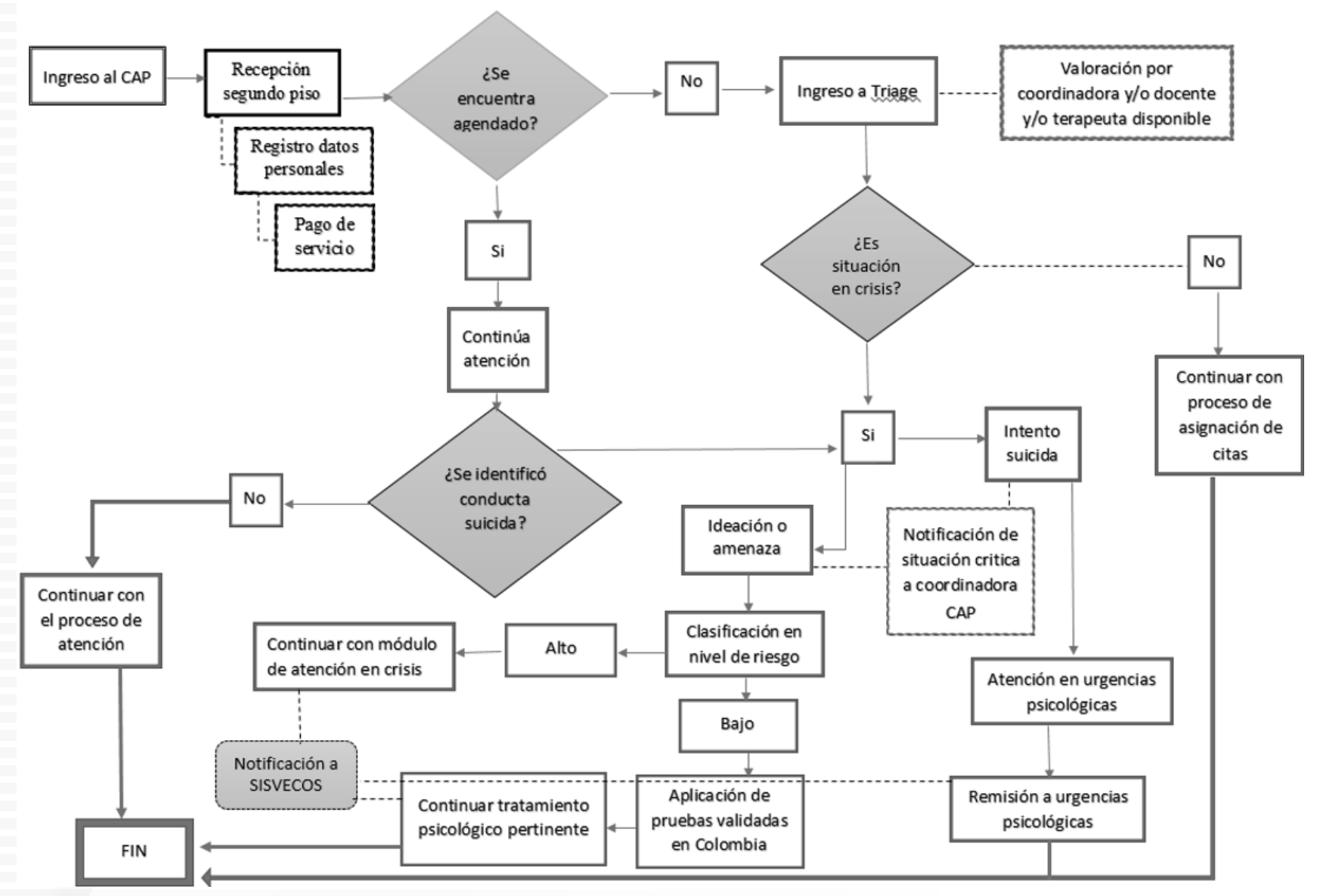

Figura 3. Flujograma de la ruta de atención diseñada.

- Módulo Atención en crisis: El objetivo de este módulo es contener la vivencia suicida, posibilitar un tratamiento psicológico posterior (terapéutico o farmacológico) y facilitar al usuario la posibilidad de exteriorizar su deseo de muerte y la comprensión de su conducta suicida. El diseño fue basado en lo propuesto por Slaikeu (1996), el cual incluye tres ideas fundamentales: intervención en crisis, conductas suicidas y estrategias terapéuticas, teniendo en cuenta la vulnerabilidad del consultante, la importancia de los factores protectores y la importancia de una intervención inmediata para lograr superar la crisis.

-Módulo Atención psicológica: Terapia cognitivo conductual dividida en 14 sesiones, donde se contempla la evaluación, intervención y seguimiento.

Fase de validación por Jueces Expertos: Se realizó una validación de contenido con 10 jueces expertos de manera individual a partir del formato diseñado para tal fin. Para la evaluación, se les pidió a los jueces calificar en una escala de 1 a 4 (suficiencia, claridad, pertinencia y relevancia) los componentes del protocolo (protocolo de activación, la ruta de atención, módulo de atención en crisis y módulo de atención psicológica). A continuación, se describen los resultados de esta fase:

Protocolo de activación: En este componente los jueces resaltan la importancia y pertinencia de estos eventos ya que permiten organizar, guiar y brindar atención al consultante al momento de llegar al centro.

Ruta de atención: Se encontró que es claro, pertinente y relevante para la problemática de ideación suicida, ya que permite a los usuarios del protocolo seguir con facilidad los pasos de la atención, recepción de caso y notificaciones al sistema de salud del país.

Módulo de atención en crisis: Los jueces consideraron que este módulo es relevante dentro del protocolo, ya que cuenta con los pasos para realizar una atención en primeros auxilios psicológicos en primera y segunda instancia, y es pertinente para problemáticas de ideación suicida. 
Módulo de intervención psicológica: Los jueces consideraron que no es suficiente para la atención de ideación suicida, ya que no permite una flexibilidad en la atención al momento de una crisis o una recaída del consultante (Ver Tabla 1).

Tabla 1

Calificaciones de los jueces expertos

Componente Suficiencia Claridad Pertinencia Relevancia

\begin{tabular}{lcccc}
\hline $\begin{array}{l}\text { Protocolo de } \\
\text { activación }\end{array}$ & 3,5 & 3,3 & 3,7 & 3,8 \\
$\begin{array}{l}\text { Ruta de } \\
\text { atención }\end{array}$ & 3,5 & 3,4 & 3,8 & 3,9 \\
$\begin{array}{l}\text { Módulo de } \\
\text { Atención en } \\
\text { crisis }\end{array}$ & 3,3 & 3,3 & 3,7 & 3,8 \\
$\begin{array}{l}\text { Módulo de } \\
\text { Intervención }\end{array}$ & 2,6 & 3 & 3,3 & 3,3 \\
\begin{tabular}{l} 
Psicológica \\
\hline \hline
\end{tabular}
\end{tabular}

Nota: El rango de calificación se encuentra entre 1 y 4.

De acuerdo con el coeficiente de razón de validez de contenido de Lawshe (1975), modificado por Tristán (2008), tres de los cuatro componentes evaluados son aceptados puesto que los valores superan el punto de corte mínimo de 0,60 (ver Tabla 2). Se observa que el componente de módulo de atención psicológica, además del concepto de los jueces, se encuentra por debajo del valor establecido, por lo tanto, se procedió a su eliminación.

Tabla 2

Coeficiente de razón de validez de contenido

\begin{tabular}{ll}
\hline \hline Componentes & IVC \\
\hline Situaciones por las cuales se active el protocolo & 0,6 \\
Ruta de atención & 0.6 \\
Módulo de atención en crisis & 0,6 \\
Módulo de atención psicológica & $-0,2$ \\
\hline \hline
\end{tabular}

\section{Discusión}

De acuerdo con Sánchez et al. (2011), el impacto que tiene la conducta suicida en la salud pública se debe al uso adecuado de los diferentes métodos y técnicas para asegurar un desempeño apropiado en esta área. El uso de protocolos clínicos tiene como fin estandarizar la calidad en la atención de salud y ayudar al clínico a tomar las mejores decisiones en momentos de incertidumbre.

Esto concuerda con el objetivo propuesto de diseñar el protocolo y validarlo para ser adoptado por el Centro de Atención Psicológica Universitaria y estandarizar cuáles son los casos que implican una conducta suicida, qué pasos seguir para el ingreso, registro, asignación de terapeuta y especialidad, notificaciones al sistema de salud del país y manejo del consultante.

Dado que a nivel internacional el NHS (2014) elaboró la guía para diseñar protocolos, el equipo de investigadores, con el propósito de tener un protocolo estandarizado a nivel internacional y en consonancia con la Guía de Práctica Clínica \# 60 sobre prevención e intervención de la conducta suicida, adoptada por el Ministerio de Salud de Colombia, utilizó algunos pasos para el diseño de la misma. Esta guía contribuyó a elaborar un protocolo ajustado a las necesidades locales y nacionales (Guía de Práctica Clínica Número 60, 2017).

El propósito de este protocolo fue cubrir una de las necesidades que se presentan actualmente en la comunidad de salud mental y que generan riesgo vital en la población, además, brindar al terapeuta un protocolo con herramientas y una ruta a seguir para una atención óptima.

Frente a las situaciones por las cuales se activa el protocolo que fueron identificadas al momento del diseño, no se encuentra en la literatura algún evento relacionado. Los factores de riesgo individuales, familiares y contextuales con los que cuenta una persona pueden activar en primera medida la ruta de atención psicológica del Centro de Atención Psicológica Universitario.

Se puede concluir que el protocolo de atención psicológica para la ideación suicida es pertinente, suficiente, claro y relevante para abordar la problemática que pretende tratar. Dado que este protocolo se usará en el Centro de Atención Psicológica Universitaria, donde hay estudiantes de posgrado de psicología clínica y de la especialización en intervención psicológica en situaciones de crisis, y de acuerdo con la GPC número 60 están facultados para realizar la atención en conducta suicida, por lo cual se recomienda la utilización del protocolo por parte de estos profesionales. Finalmente, se recomienda 
completar los pasos de la guía NHS (2014), como son: la capacitación, actualización y monitoreo de este protocolo en un periodo de tiempo de un año.

\section{Referencias}

Beaglehole, R., Bonita, R., \& Kjellstróm, T. (2008). Epidemiología Básica. Washington D. C.: Organización Panamericana de la Salud.

Guía de Práctica Clínica para la prevención, diagnóstico y tratamiento de la ideación y/o conducta suicida (Adopción). (2017). Sistema General de Seguridad Social en Salud, Colombia. Guia Número 60.

Instituto Colombiano de Medicina Legal y Ciencias Forenses. (2018). Forensis, Datos para la Vida. Bogotá. ISSN: 2145-0250. Recuperado de http://www. medicinalegal.gov.co/documents/20143/262076/ Forensis + 2017+ Interactivo.pdf/0a09fedb-f5e811f8-71ed-2d3b475e9b82.

Ministerio de la Protección Social. (2006). Ley número 1090, Congreso de Colombia. Recuperado de http://www.sociedadescientificas.com/userfiles/ file/LEYES/1090\%2006.pdf

Ministerio de Salud. (2018). Boletín de salud mental Conducta suicida. Subdirección de Enfermedades No Transmisibles.

Ministerio de Salud y Protección Social. (2018). Resolución Número 4886. Recuperado de https:// www.minsalud.gov.co/Normatividad_Nuevo/ Resoluci\%C3\%B3n\%20No.\%204886\%20de\%20 2018.pdf
Ministerio de Salud y Protección Social. (2018). Vigilancia en salud pública. Recuperado de https://www. minsalud.gov.co/salud/publica/epidemiologia/Paginas/vigilanciasaludpublica.aspx

Ministerio de Salud y Protección Social y Colciencias. (2015). Encuesta Nacional de Salud Mental 2015, tomo I.

Montero, I., \& León, O. (2007). A guide for naming research studies in Psychology. International Journal of Clinical and Health Psychology, 7(3), 847-862.

Organización Mundial de la Salud. (2018). Temas de salud: Epidemiología. Recuperado de https://www.who. int/topics/epidemiology/es/

Resolución número 8430. (1993). Ministerio de Salud, Colombia. Recuperado de https://www.minsalud. gov.co/sites/rid/Lists/BibliotecaDigital/RIDE/DE/ DIJ/RESOLUCION-8430-DE-1993.PDF

Sánchez, Y., González, J., Molina, O., \& Guil, M. (2011). Guía para la elaboración de protocolos. Biblioteca Lascasas, 7(1). Recuperado de http://www.index-f. com/lascasas/documentos/lc0565.php

Slaikeu, K. (1996). Intervención en crisis. Manual para práctica en investigación. Bogotá: Manual Moderno.

Saura, L., \& Saturno, P. (1996). Clinical Protocols: How are Constructed? Proposal of a Model for its Design and Elaboration. Evaluation and Improvement of Clinical Protocols Group. Atención Primaria, 18(2). 91-96

Tristán, A. (2008). Modificación al Modelo de Lawshe para el Dictamen Cuantitativo de la Validez de Contenido de un Instrumento Objetivo. Avances en Medición, 6. 37-48. 